\title{
Automatically Computed ECG Algorithm for the Quantification of Myocardial Scar and the Prediction of Mortality
}

Patrick Badertscher, $\mathrm{MD}^{1 *}$; Ivo Strebel, $\mathrm{MS}^{1 *}$; Ursina Honegger, MS ${ }^{1}$; Nicolas

Schaerli, MD; Deborah Mueller, MD; Christian Puelacher, MD'; Max Wagener, MD;

Roger Abächerli, MD¹,2; Joan Walter, BSc ${ }^{1}$; Zaid Sabti, MD'; Lorraine Sazgary, MD;

Stella Marbot, MD¹; Jeanne du Fay de Lavallaz MD¹; Raphael Twerenbold, MD ${ }^{1,3}$; Jasper Boeddinghaus, MD; Thomas Nestelberger, MD¹; Nikola Kozhuharov, MD;

Tobias Breidthardt, MD ${ }^{1,4}$; Samyut Shrestha, MD; Dayana Flores, MD¹; Carmela

Schumacher, MSc${ }^{1}$; Damian Wild, MD³; Stefan Osswald, MD¹; Michael J. Zellweger,

$$
\mathrm{MD}^{1} \text {; Christian Mueller, MD'; Tobias Reichlin, } \mathrm{MD}^{1}
$$

${ }^{1}$ Cardiovascular Research Institute Basel (CRIB) and Division of Cardiology, University Hospital Basel, University of Basel, Switzerland; ' 2 Insitute for Medical Engineering (IMT),Lucerne University of Applied Sciences and Arts, Horw, Switzerland; ${ }^{3}$ University Heart Center Hamburg, Clinic for General and Interventional Cardiology, Hamburg, Germany; ${ }^{4}$ Department of Internal Medicine, University Hospital

Basel, University of Basel, Switzerland; ${ }^{5}$ Division of Nuclear Medicine, University Hospital Basel, University Basel, Switzerland

*both authors have contributed equally to this analysis and should please be considered co-first authors

Short Title: Automated ECG Scar Quantification and Outcomes in Heart Failure

Key words: Selvester QRS-score, ECG, myocardial scar, heart failure, cardiac imaging

Trial-Registration: www.clinicaltrials.gov. Identifier, NCT01838148 and NCT01831115

Word Count: 4250

\section{Correspondence to:}

PD Dr. Tobias Reichlin

Department of Cardiology. University Hospital Basel

Petersgraben 4. $\mathrm{CH}-4031$ Basel. Switzerland.

Phone Number: +4161556 58 23. Fax number: +4161265 4598

E-mail: tobias.reichlin@usb.ch 


\section{Abstract (250)}

Background: Myocardial scar is associated with adverse cardiac outcomes. The Selvester QRSscore was developed to estimate myocardial scar from the 12-lead ECG, but its manual calculation is difficult. An automatically computed QRS-score would allow identification of patients with myocardial scar and an increased risk of mortality.

Objectives: To assess the diagnostic and prognostic value of the automatically computed QRSscore.

Methods: The diagnostic value of the QRS-score computed automatically from a standard digital 12-lead was prospectively assessed in 2'742 patients with suspected myocardial ischemia referred for myocardial perfusion imaging (MPI). The prognostic value of the QRS-score was then prospectively tested in 1'151 consecutive patients presenting to the emergency department (ED) with suspected acute heart failure (AHF).

Results: Overall, the QRS-score was significantly higher in patients with more extensive myocardial scar: the median QRS-score was 3 (IQR 2-5), 4 (IQR 2-6), and 7 (IQR 4-10) for patients with $0 \%, 5-20 \%$ and $>20 \%$ myocardial scar as quantified by MPI $(p<0.001$ for all pairwise comparisons). A QRS-Score $\geq 9(n=284,10 \%)$ predicted a large scar defined as $>20 \%$ of the LV with a specificity of $91 \%(95 \% \mathrm{Cl} 90-92 \%)$. Regarding clinical outcomes in patients presenting to the ED with symptoms suggestive of AHF, mortality after 1 year was $28 \%$ in patients with a QRSscore $\geq 3$ as opposed to $20 \%$ in patients with a QRS-score $<3(p=0.001)$.

Conclusions: The QRS-Score can be computed automatically from the 12-lead ECG for simple, non-invasive and inexpensive detection and quantification of myocardial scar and for the prediction of mortality. 


\section{Introduction}

Myocardial scar is associated with heart failure and worse outcome. ${ }^{1}$ Myocardial scar also provides the substrate for reentrant ventricular arrhythmias and sudden cardiac death. ${ }^{2}$ In order to improve survival and reduce morbidity, better strategies for identification and risk stratification of patients with heart failure and myocardial scar are an unmet clinical need. ${ }^{3}$

The gold standard for identification and quantification of myocardial scar are cardiac imaging techniques such as cardiovascular myocardial resonance imaging $(\mathrm{CMR})^{4}$ or myocardial perfusion imaging $(\mathrm{MPI})^{5}$. However, these techniques have relevant limitations as risk stratification tools, since they are neither bedside tests nor widely available due to the costs and clinical expertise required. Preferably, an initial screening should involve simpler, cheaper and universally available tests, that may empower general practitioners and reduce the need for specialist referral, which could help to decrease the current fragmentation in clinical care.

The standard 12-lead ECG is a universally available, inexpensive test that would fulfill these requirements. Traditionally, myocardial scar was assessed qualitatively in the 12-lead ECG focusing on the presence of Q-waves. ${ }^{6}$ More recently, the Selvester QRS-score was developed based on computer simulation. ${ }^{7,8}$ The idea of the scoring system is to detect and quantify myocardial scar from the 12-lead ECG by measuring changes in Q-, R-, and S-wave duration and amplitudes and morphologies. ${ }^{7,8}$ Later, modified criteria were suggested to be used in the presence of ECG confounders such as bundle branch blocks or LV hypertrophy. ${ }^{9}$ In patients with left ventricular (LV) systolic dysfunction and heart failure, the extent of myocardial scar as assessed by the QRS-score has been shown to predict survival and the progression of symptoms. ${ }^{10,11}$ Manual calculation of the QRS-score is difficult, tedious and may take up to 15 minutes per ECG, ${ }^{12}$ thereby limiting its application in clinical practice. This problem could be overcome by the development of automated algorithms for calculation of the QRS-score using digital ECG data. ${ }^{13,14}$ However, to the best of our knowledge, the automated QRS score and its 
association with the extent of myocardial scar has never been prospectively validated against an imaging gold-standard.

The simple, non-invasive, and inexpensive detection and quantification of myocardial scar would be particularly helpful in the management of patients presenting with the two cardinal symptoms related to cardiac disease: chest discomfort and suspected myocardial ischemia as well as shortness of breath and suspected acute heart failure. We therefore performed a diagnostic study in two distinct large cohorts to examine the diagnostic and prognostic value of the QRS-score. Our aim was to determine (a) the association of the Selvester QRS-score automatically computed from the standard 12-lead ECG with the extent of myocardial scar as quantified by myocardial perfusion imaging (MPI), and (b) the prognostic value of the QRS-score to predict all-cause mortality during long-term follow-up in patients presenting to the ED with shortness of breath. 


\section{Methods}

\section{Patient Population Myocardial Perfusion Imaging Cohort: BASEL VIII Study}

We prospectively enrolled consecutive patients referred to the University Hospital Basel for the evaluation of suspected myocardial ischemia by rest/stress myocardial perfusion imaging (MPI) for the "Biomarkers and ECG signals in exercise-induced myocardial ischemia (BASEL VIII)" study (ClinicalTrials.gov registry, number NCT01838148). ${ }^{15,16}$ The authors designed the study, gathered, and analysed the data according to the STARD guidelines for studies of diagnostic accuracy (Supplemental Methods).

\section{MPI acquisition and interpretation protocol}

Patients underwent routine rest/stress imaging using either a dual isotope (201TI for rest, 99mTc sestamibi for stress) MPI protocol or a protocol with 99Tc for both examinations ${ }^{17}$. A standardized, stepwise, and symptom-limited bicycle exercise stress testing or a pharmacological stress testing protocol was performed..$^{18} \mathrm{MPI}$ were evaluated by an expert team consisting of a cardiologist and a nuclear medicine physician and scored semi-quantitatively using a 17 segment bull's eye scheme with a 5 -point scale $(0=$ normal. $1=$ mildly reduced tracer uptake. $2=$ moderately reduced tracer uptake. $3=$ severely reduced tracer uptake. $4=$ no uptake) ${ }^{18}$ Summed stress scores (SSS) and summed rest scores (SRS) were computed by adding the scores of the 17 segments in the stress and rest images respectively. Left ventricular ejection fraction (LVEF) was computed from post-stress gated images. The adjudication of the presence or absence of myocardial scar was based on the number of non-reversible segments (stress-rest score combinations of 4-4, 4-3, 3-3, 3-2, or 2-2) was summed up and used as a surrogate of the extent of myocardial scar size as described previously, with each non-reversible segment representing approximately $5 \%$ of myocardial scar. ${ }^{19}$

\section{Patient Population Acute Decompensated Heart Failure Cohort: BASEL V Study}

We prospectively enrolled patients with symptoms suggestive of acute heart failure (AHF) presenting to the ED of the University Hospital Basel for the "Basics in acute shortness of breath 
evaluation (BASEL V)" study (ClinicalTrials.gov registry, number NCT01831115). ${ }^{20,21}$ A final diagnosis of AHF was adjudicated by two independent cardiologists using all available information pertaining to the individual patient after hospital discharge applying the guidelines of the European Society of Cardiology ${ }^{3}$. In case of disagreement, the case was reviewed by a third cardiologist who decided ultimately. Enrollment was independent of renal function, but patients with terminal renal failure on chronic renal replacement therapy were excluded for this analysis.

\section{Follow-Up BASEL V Study}

Patients were contacted after 1 year by telephone calls or in written form. Furthermore, information was obtained by institutional chart review, the family physician's records and the national registry on mortality. The primary prognostic endpoint was all-cause mortality during 1 year of follow-up.

\section{Ethic's approval}

BASEL VIII and BASEL V were carried out according to the principles of the Declaration of Helsinki and approved by the local ethics committee. Written informed consent was obtained from all participating patients.

\section{Recording of digital ECGs}

Resting 12-lead ECGs were acquired prior to the exercise stress test (BASEL VIII) or during standard clinical assessment of patients in the ED (BASEL V) using an AT-110 or CS-200 Excellence ECG device (Schiller AG, Baar, Switzerland). The digital ECG raw data was recorded using a minimal sampling rate of $500 \mathrm{~Hz}$, a minimal resolution of $5 \mu \mathrm{V} / \mathrm{bit}$ and a minimal diagnostic signal bandwidth of $0.05 \mathrm{~Hz}$ to $150 \mathrm{~Hz}$.

\section{Principle of the Selvester QRS-score to quantify myocardial scar from the 12-lead ECG}

The Selvester QRS-score analysis protocol to quantify myocardial scar from the 12-lead ECG has previously been reported in detail. ${ }^{9,22}$ In brief, ECGs are first classified for confounders into the 6 categories: left bundle branch block (LBBB), right bundle branch block (RBBB) and left anterior fascicular block (LAFB), isolated RBBB, isolated LAFB, left ventricular hypertrophy (LVH) or no 
confounders. Second, points for Q-, R-, and S-wave amplitudes, durations, amplitude ratios, and notches in 10 of the 12 standard ECG leads (excluding leads III and aVR) are awarded according to the ventricular conduction/hypertrophy type. With a total of 54 criteria, a maximum QRS-score of 32 points can be reached, with each point representing $3 \%$ of the LV with myocardial scar. In addition, age and sex adjustments are made to the score criteria, as the normal limits of Q-, R-, and S-wave amplitudes and durations may differ by age and sex. For illustration and better understanding of the Selvester QRS-score, a sample scoring sheet and additional details are provided within the online supplemental appendix.

\section{Automated calculation of the Selvester QRS-score}

The raw data of the digital 12-lead ECG was analyzed using the Schiller ECG analysis program (ETM V01.12.09.00, Schiller AG, Baar, Switzerland). Automated Q-, R-, and S-amplitude and duration measurements along with diagnostic statement codes were extracted and used for analysis. The criteria used to automatically classify ECG confounder types for QRS scoring have been published before ${ }^{14}$ and are listed in the online supplemental appendix.

\section{Statistical analysis}

Continuous variables are presented as mean (standard deviation) or median (interquartile range [IQR]); categorical variables, as numbers and percentages. Differences in baseline characteristics between patients were assessed using the Mann-Whitney test for continuous variables and the Pearson Chi Square test for categorical variables. Receiver-operating-characteristic (ROC) curves were constructed to assess the diagnostic accuracy of the QRS-score to diagnose a myocardial scar as adjudicated based on MPI. Survival according to QRS-score categories was plotted in Kaplan-Meier curves. The log-rank test was used to assess differences in mortality between groups. We used univariate Cox proportional hazard analysis to compute hazard ratios and $95 \%$ confidence intervals of the QRS-score to predict all-cause mortality and cardiovascular rehospitalization. The model was further adjusted for the following established predictors of death to assess for independent prognostic value of the QRS-score (with dichotomous cut-points selected 
where appropriate): age $\geq 70$, gender, dyspnea NYHA IV vs. II and III vs. II, QRS duration $\geq$ $120 \mathrm{~ms}$, creatinine $\geq 105 \mathrm{umol} / \mathrm{l}$, heart rate $\geq 80 \mathrm{bpm}$ and the presence of diabetes mellitus, atrial fibrillation or LBBB.

All hypothesis testing was two-tailed and p-values $<0.05$ were considered statistically significant. Statistical analyses were performed using IBM SPSS Statistics for Windows, version 22.0 (SPSS Inc, Chicago, IL) and the R statistical package (MathSoft, Seattle, WA). 


\section{Results}

\section{Enrolment and characteristics of patients referred for myocardial perfusion imaging}

(BASEL VIII Study)

From January 2010 to November 2014, a total of 4'219 unselected patients undergoing evaluation of suspected exercise inducible myocardial ischemia by myocardial perfusion imaging (MPI) were prospectively enrolled. Patients without a digital 12-lead ECG were excluded from analysis $\left(n=1^{\prime} 002\right)$, as were patients with non-specific bundle branch block (NBBB, $\left.n=99\right)$ and ventricular pacing $(n=57)$, which precluded automated calculation of the QRS-Score. Furthermore, patients with no SRS-score available, meaning patients with only stress imaging $(n=319)$ were excluded, leaving 2'742 patients for analysis (Supplemental Figure 1).

Baseline characteristics of the patients are shown in Table 1. 32\% of the patients had a history of myocardial infarction (MI) with a median time interval from MI to enrolment of 3.4 years (IQR 0.5310.5 years).

Overall, no evidence of myocardial scar as quantified by MPI was seen in $62 \%$ of the patients. In $32 \%$ of the patients, the extent of myocardial scar was $5-20 \%$ and in $6 \%$ of the patients, more than $20 \%$ myocardial scar was present.

\section{Levels of the QRS-score in the MPI cohort}

The median QRS-score was 3 (IQR 2-6), representing an estimated LV scar of 9\% (IQR 6-18\%). Baseline characteristics of the patients stratified for QRS-scores $\geq$ or $<$ the median QRS-score of 3 are shown in Table 1. Patients with a QRS-score $\geq 3$ more often had a history of coronary artery disease (CAD), MI and revascularization. The left ventricular ejection fraction (LVEF) as measured by MPI was significantly lower in patients with higher QRS-scores (all p-values < 0.001).

\section{Association of the QRS-score with the extent of myocardial scar}

As shown in Figure 1, the QRS-score was significantly higher in patients with more extensive myocardial scar as quantified by MPI: for patients without myocardial scar, the median QRS-score 
was 3 (IQR 2-5), for patients with 5-20\% myocardial scar, the median QRS-score was 4 (IQR 2-6), and for patients with more than $20 \%$ myocardial scar, the QRS-score was 7 (IQR 4-10, p $<0.001$ for all pairwise comparisons). Of all patients with myocardial scar, 89\% had a QRS-score $\geq 1$, resulting in a sensitivity of $94 \%(95 \% \mathrm{Cl} 90-97 \%)$ for the detection of any myocardial scar. Diagnostic accuracy of the QRS-score for the detection of any myocardial scar as quantified by the AUC was $0.60(95 \% \mathrm{Cl} 0.58-0.62)$. For the detection of a myocardial scar $>20 \%$ the AUC was $0.73(95 \% \mathrm{Cl} 0.69-0.78)$. A QRS-Score $\geq 9(n=284,10 \%)$ predicted a large scar defined as $>20 \%$ of the LV with a specificity of $91 \%(95 \% \mathrm{Cl} 90-92 \%)$.

\section{Impact of ECG confounders on the association with myocardial scar}

The ECG was free of confounders in $82 \%$ of patients. Of those with ECG confounders, complete LBBB was present in $3.4 \%$, combined RBBB \& LAFB in $2.2 \%$, isolated RBBB in $3.2 \%$, isolated LAFB in $3.0 \%$, and LVH in $5.8 \%$ of all cases. Presence and type of ECG confounders of cardiac de- and repolarization resulted in significant changes of the QRS-score. Median QRS-scores for the different ECG confounder groups overall and according to the extent of myocardial scar are shown in Table S1. Of particular note, the presence of LAFB either in isolation or in conjunction with RBBB resulted in higher QRS-scores compared to all other groups.

\section{Diagnostic value of the QRS-score to detect a reduced LVEF $<35 \%$}

A reduced LVEF $<35 \%$ was found in 209 patients (8\%). As shown in Figure 2, the QRS-score was significantly higher in patients with reduced LVEF: for patients with LVEF $<35 \%$, the median QRSscore was 5 (IQR 3-9) and for patients with more than 55\% LVEF, the QRS-score was 3 (IQR 1-5, $p<0.001)$. For patients with a QRS-score $\geq 9(10 \%)$, the specificity to detect a reduced LVEF was $91 \%(95 \% \mathrm{Cl} 90-92 \%)$ and sensitivity was $24 \%(95 \% \mathrm{Cl} 19-31 \%)$. On the other hand, a QRS-score of $0(12 \%)$ had a sensitivity of $94 \%(90-97 \%)$ and a specificity of $13 \%(95 \% \mathrm{Cl} 12-14 \%)$ to rule-out a reduced $L V E F<35 \%$. 
Enrolment and characteristics of patients with symptoms suggestive of AHF (BASEL V

\section{Study)}

From September 2001 to January 2013, a total of 2'130 patients with symptoms suggestive of AHF presenting to the ED were prospectively enrolled. Patients without a digital 12-lead ECG were excluded from analysis $(n=881)$, as were patients with NBBB $(n=84)$ and ventricular pacing $(n=14)$, leaving 1'151 patients for analysis (Figure S2). Overall, $61 \%$ of patients had a final adjudicated diagnosis of AHF.

\section{Levels of the QRS-score in the AHF cohort}

Overall, the median QRS-score was 3 (IQR 1-6) equal to an estimated LV scar of 9\% (3-18\%). Baseline characteristics of the patients stratified for QRS-score levels $\geq$ or $<$ the median value of 3 are shown in Table 2. Similarly, to the Basel VIII cohort, patients with higher QRS-score had more often a history of CAD, MI and revascularization and had significantly higher B-type natriuretic peptide (BNP) levels at presentation (345 vs $605 \mathrm{ng} / \mathrm{l}, \mathrm{p}<0.001)$. A QRS-score $\geq 3$ was associated with a higher probability for a final adjudicated diagnosis of AHF (67\% vs. $55 \%$. $p<0.001)$

\section{Diagnostic value of the QRS-score to detect a reduced LVEF $<35 \%$ in patients with}

\section{suspected AHF}

The LVEF was assessed by transthoracic echocardiography (TTE) in 653 patients. A reduced LVEF $<35 \%$ was found in $21 \%$ of the patients. As shown in Figure 2, the QRS-score was significantly higher in patients with reduced LVEF: for patients with LVEF $<35 \%$, the median QRSscore was 4 (IQR 2-7), for patients with 35-55\% LVEF, the median QRS-score was 3 (IQR 1-6), and for patients with more than 55\% LVEF, the QRS-score was 2 (IQR 1-5, p < 0.001).

Similar to the results in the BASEL VIII cohort, a QRS-score $\geq 9(11 \%)$ had specificity to detect a reduced LVEF by TTE of $91 \%(95 \% \mathrm{Cl} 89-94 \%)$ with a corresponding sensitivity of $20 \%(95 \% \mathrm{Cl}$ $14-28 \%)$. On the other hand, a QRS-score of $0(18 \%)$ had a sensitivity of $89 \%(95 \% \mathrm{Cl} 82-94 \%)$ and a specificity of $16 \%(95 \% \mathrm{Cl} 13-20 \%)$ to rule-out a reduced LVEF $<35 \%$. 


\section{Prognostic value of the QRS-score for the prediction of mortality during follow-up}

During a mean follow-up time of 430 days, there were 367 deaths (32\%) in the whole cohort. Allcause mortality after 1 year was $28 \%$ in patients with a QRS-score $\geq 3$ as opposed to $20 \%$ in patients with a QRS-score $<3$ in Kaplan-Meier analysis ( $p=0.001$ Figure 3A). The difference in mortality could be observed as early as after 30 days with mortality rates of $9 \%$ vs. $5 \%$ in patients with a QRS-score $\geq 3$ vs. $<3(p=0.02)$. When assessing only patients with a final adjudicated diagnosis of AHF (61\% of the overall cohort) the all-cause mortality rates at 1-year follow-up were $32 \%$ vs. $23 \%$ for patients with a QRS-score $\geq 3$ and $<3(p=0.01)$. The observation of higher mortality rates at 30 and 360 days in patients with a QRS-score $\geq 3$ was confirmed in patients with and without ECG confounders present in the 12-lead ECG (Table S2). In univariable cox proportional hazard analysis QRS-score as a continuous variable (per 3-point increase) was significantly related with all-cause mortality (Hazard Ratio, 1.11, 95\% Cl 1.02-1.22, $p=0.02$ ). When using a dichotomous cut-off for presence of scar defined by the median QRS-score of $\geq 3$, there were $48 \%$ more deaths compared to patients with a QRS-score $<3$ (Hazard Ratio 1.48, 95\% Cl 1.16-1.89, $\mathrm{p}=0.001$ ). After adjusting for important clinical factors (including age, diabetes mellitus, dyspnea and renal failure) and ECG parameters (QRS duration, heart rate, atrial fibrillation and LBBB) in multivariable analysis, a QRS-score $\geq 3$ remained an independent predictor of mortality (Hazard Ratio 1.42, 95\%Cl 1.10-1.83, for QRS >=3 versus <3, $p=0.006$, Table 3).

\section{QRS-score association with cardiovascular re-hospitalization}

During follow-up, a total of 248 patients (22\%) required a re-hospitalization due to congestive heart failure $(\mathrm{CHF})$ or another cardiac reason. There were $45 \%$ more re-hospitalizations in patients with a QRS-score $\geq 3(\mathrm{HR} 1.45,95 \% \mathrm{Cl} 1.12-1.89, \mathrm{p}=0.005$, Figure 3B). The QRS-score as a continuous variable (per 3 points increase) was also significantly related with first occurrence of rehospitalization during 360 days $(\mathrm{HR} 1.18,95 \% \mathrm{Cl} 1.08-1.30, \mathrm{p}=0.001)$. After adjusting for clinical factors and ECG parameters, a QRS-score $\geq 3$ was an independent predictor of re-hospitalization 
along with the presence of atrial fibrillation, diabetes mellitus and renal failure at presentation (Table S3). 


\section{Discussion}

In two large prospective diagnostic studies, we aimed (a) to assess the association of the QRSscore automatically computed from the 12-lead ECG with the extent of myocardial scar as quantified by MPI in 2742 patients and (b) to study the prognostic value of the QRS-score to predict mortality during long-term follow-up in 1151 patients presenting to the ED with symptoms suggestive of AHF.

We report three major findings: First, QRS-scores at presentation were significantly higher in patients with more extensive myocardial scar on MPI. The diagnostic accuracy of the QRS-score for the detection of any myocardial scar on MPI as quantified by the AUC was 0.60 and increased to 0.73 for the detection of $>20 \%$ myocardial scar. Second, higher QRS-scores predicted a reduced LVEF in both cohorts. A QRS-score $\geq 9$ had a specificity of $91 \%$ in both cohorts to detect a LVEF $<35 \%$. Third, the QRS-score was a powerful predictor of all-cause mortality and rehospitalization in patients presenting to the ED with symptoms suggestive of AHF independent of important other clinical and ECG factors. All-cause mortality after 1 year was $28 \%$ in patients with a QRS-score $\geq 3$ as opposed to $20 \%$ in patients with a QRS-score $<3(p<0.001)$.

These findings extend and corroborate previous data on the possible clinical utility of the QRSscore to predict the presence of myocardial scar..$^{13,23-27}$ This study has important methodological strengths differentiating it from previous studies on the QRS-score, including but not limited to its prospective design, the automated calculation of the updated QRS scoring system, the applicability in the presence of traditional ECG confounders and the use of the adjusted criteria for age and sex. Furthermore, to the best of our knowledge, this is the largest prospective validation studying the association of the QRS-score with the extent of myocardial scar as quantified by a cardiac imaging technique.

The diagnostic performance of the QRS-score to detect myocardial scar in this study was similar to that reported elsewhere using CMR. ${ }^{13,23-25,27}$ Previously, a wide range of diagnostic accuracy to detect myocardial scar was shown with AUC between 0.62 and $0.91 .^{13,23,27}$ The reasons for this 
variety are manifold. First, in the early beginning, Selvester and Coworkers ${ }^{8,28}$ developed the QRS-score in the non-reperfusion era for acute $\mathrm{MI}$ in comparison to post-mortem histopathological analysis. It is therefore possible that the score best reflects acute scar resulting from nonreperfused fatal myocardial infarctions rather than chronic, remodelled myocardial scar late after AMI. This hypothesis was further supported by CMR studies, as also stronger correlation for ECG estimated scar was seen in patients early after a first MI. ${ }^{24,26}$ Second, the association between the QRS-score and the extent of myocardial scar on MPI is more robust in patients without ECG confounders. Hence, the diagnostic performance of the QRS score varies depending on the prevalence of ECG confounders in a cohort. This proportion of ECG confounders was $18 \%$ in our study, and remarkably higher compared to some of the previous studies, which enrolled as little as 9 patients $(6 \%)$ with ECG confounders. ${ }^{29}$ With regards to the different subgroups of ECG confounders, we could confirm the previous observation that the algorithm overestimated the extent of myocardial scar particularly in patients with isolated LAFB or LAFB \& RBBB. ${ }^{13,27}$ As opposed to the QRS-score in patients without ECG confounders, the modified QRS scoring system in the presence of conduction defects was created based on computer simulation and has never been validated with post-mortem analysis. ${ }^{9}$ Given that the presence of LAFB significantly affects leads $\mathrm{V}_{4}-\mathrm{V}_{6}$, Wieslander et al. ${ }^{27}$ made an attempt to modify the scoring system by excluding points derived from leads $V_{4}-V_{6}$ in LAFB patients, which improved the $A U C$ for scar detection from 0.62 to 0.67 . Third, the manual calculation of the QRS-score is difficult, tedious and may take up to 15 minutes per ECG. ${ }^{12}$ Accordingly, results were often highly operator-dependent and its application in clinical practice was limited. Using an automated algorithm for calculation of the QRS-score from digital ECG data, the method is much more robust and can be easily applied in clinical practice. ${ }^{14,30}$ Our study is the first to prospectively validate an automatically computed QRS-score against an imaging gold standard. The similar diagnostic accuracy compared to studies using manual calculation done by extensively trained and highly experienced ECG readers 
is important and supports the suitability of the automated QRS score for a broad clinical application. ${ }^{13,23,27}$

The extent of myocardial scar is correlated with LVEF. Accordingly, higher QRS-scores predicted a reduced LVEF and a QRS-score $\geq 9$ had a specificity of $>90 \%$ in both cohorts to detect a LVEF $<35 \%$. Given that current guidelines for ICD-implantation for primary prevention of sudden cardiac death focus predominantly on a LVEF $<35 \%$, the QRS-score may serve as a simple screening tool in general medical populations to identify patients potentially at risk that might benefit from further work-up with echocardiography.

Beyond its diagnostic utility in the detection of myocardial scar, the QRS-score is a simple, inexpensive and easily implementable method for the prediction of clinical outcome. In our cohort of patients presenting with symptoms suggestive of AHF, those with a QRS-score above the median ( $\geq 3$ ) had a $48 \%$ higher risk of mortality and a $45 \%$ higher risk of re-hospitalization due to cardiovascular causes within 1-year after the index presentation. This is in line with previous observations made in studies investigating the prognostic value of the QRS-score in various other settings ${ }^{14,23,26,31,32}$. Similar to our results, a recent study showed that in 100 consecutive acute MI patients, a QRS-score $\geq 3$ at hospital discharge was associated with significantly more deaths or hospitalizations for heart failure during follow-up compared with a QRS-score $<3$ (45\% vs. $8 \%$, p < 0.001). ${ }^{32}$ It is important to highlight that the incremental value of the QRS-score for risk stratification was independent of other important clinical and ECG factors including age and QRS duration.

It has to be mentioned that the QRS-score should not be seen as an alternative to imaging techniques. Rather, it should be used as an easily available additional screening tool for risk stratification in conjunction with all available other information. Particularly in the primary care setting, the QRS-score provides a simple, non-invasive and inexpensive tool for the general practitioner for the identification of patients with so far undetected myocardial scar and an increased risk. A QRS-score $\geq 9$ in a patient with no known cardiac history should trigger referral 
to a cardiologist for additional workup. Doing so, it is a tool that may empower general practitioners in the delineation of a personalized management plan. It is possible that a further improvement of its performance can be achieved by refinement of some of the less specific criteria of the score (54 in total). ${ }^{27}$ Our results also demonstrate that research focusing on advanced ECG technologies has the potential to remarkably increase the value of the surface 12-lead ECG in clinical cardiology. ${ }^{16,33}$

Potential limitations of the present study merit consideration. First, it is a matter of ongoing discussion, which cardiac imaging modality provides the most favorable characteristics for the detection and quantification of a myocardial scar. ${ }^{34}$ While CMR provides higher spatial resolution and is therefore superior in the detection of very small myocardial scars, ${ }^{35}$ it cannot be applied in patients with pacemakers and defibrillators, and is challenging to apply in patients with claustrophobia. As MPI has important logistic advantages compared to CMR, and as a direct comparison of MPI versus CMR using absolute scar quantification with necropsy as reference standard showed comparable performance of both techniques, MPI seemed well suited for the purpose of the current study using a semi-quantitative assessment of the extent of myocardial scar. ${ }^{34}$ Second, the value of the QRS-score to predict mortality was assessed in ED patients with symptoms suggestive of AHF in our study. Further studies are needed to assess the prognostic value of the QRS-score in other patient populations, e.g. for risk stratification in the general population or in patients after AMI. Third, further research may improve the classification of borderline ECGs, e.g. the fine-tuning of the definition of LBBB. ${ }^{36}$ Fourth, it is possible that beat-tobeat variation in the QRS complex may lead to different numbers of points to be assigned to a lead depending on which beat is evaluated. In order to limit bias and error, we used a computergenerated median beat.

In conclusion, the QRS-Score, a marker quantifying myocardial scar, can be automatically computed from the 12-lead ECG. It is associated with the extent of myocardial scar as quantified by MPI. In addition, the QRS-score independently predicts all-cause mortality in patients 
presenting with symptoms suggestive of AHF and helps categorizing patients into those with reduced and normal ejection fraction. Therefore, the QRS-score provides a simple, non-invasive and inexpensive tool for the identification of patients at risk and thereby may be an important contributor in the delineation of a personalized management plan. 


\section{Acknowledgements}

The authors thank the patients who participated in the study and the staff of the Department of Nuclear Medicine.

\section{Funding}

This study was supported by research grants from the Swiss National Science Foundation, the Swiss Heart Foundation, the Cardiovascular Research Foundation Basel, the University Hospital Basel, Abbott. and BRAHMS.

\section{Disclosures}

Dr. Mueller has received research support from the Swiss National Science Foundation, the Swiss Heart Foundation, the Cardiovascular Research Foundation Basel, Abbott, Beckman Coulter, BRAHMS, Roche, Siemens. and the University Hospital Basel, as well as speaker honoraria from Abbott, ALERE, Astra Zeneca, BG Medicine, Biomerieux, Brahms, Cardiorentis, Lilly, Novartis, Roche, and Siemens. Dr. Reichlin has received research grants from the Goldschmidt-Jacobson Foundation, the Swiss National Science Foundation (PASMP3-136995) the Swiss Heart Foundation, the Professor Max Cloëtta Foundation, the Cardiovascular Research Foundation Basel, the University of Basel and the University Hospital Basel as well as speaker honoraria from

Brahms and Roche. Dr. Twerenbold has received research support from the Swiss National Science Foundation (P300PB-167803/1) and speaker honoraria/consulting honoraria from Roche, Abbott, Siemens and Brahms. Dr. Boeddinghaus has received speaker honoraria from Siemens. All other authors declare that they have no conflict of interest with this study. 


\section{References}

1. Levy D, Kenchaiah S, Larson MG, et al. Long-Term Trends in the Incidence of and Survival with Heart Failure. N Engl J Med. 2002;347(18):1397-1402. doi:10.1056/NEJMoa020265.

2. de Bakker JM, van Capelle FJ, Janse MJ, et al. Reentry as a cause of ventricular tachycardia in patients with chronic ischemic heart disease: electrophysiologic and anatomic correlation. Circulation. 1988;77(3).

3. Ponikowski P, Voors AA, Anker SD, et al. 2016 ESC Guidelines for the diagnosis and treatment of acute and chronic heart failure: The Task Force for the diagnosis and treatment of acute and chronic heart failure of the European Society of Cardiology (ESC)Developed with the special contribution of. Eur Heart J. 2016;37(27):2129-2200. doi:10.1093/eurheartj/ehw128.

4. Schmidt A, Azevedo CF, Cheng A, et al. Infarct Tissue Heterogeneity by Magnetic Resonance Imaging Identifies Enhanced Cardiac Arrhythmia Susceptibility in Patients With Left Ventricular Dysfunction. Circulation. 2007;115(15):2006-2014. doi:10.1161/CIRCULATIONAHA.106.653568.

5. Barbagelata A, Di Carli MF, Califf RM, et al. Electrocardiographic infarct size assessment after thrombolysis: insights from the Acute Myocardial Infarction STudy ADenosine (AMISTAD) trial. Am Heart J. 2005;150(4):659-665. doi:10.1016/j.ahj.2004.10.014.

6. Horan LG, Flowers NC, Johnson JC. Significance of the Diagnostic Q Wave of Myocardial Infarction. Circulation. 1971;43(3).

7. Selvester RH, Kalaba R, Collier CR, Bellman R, Kagiwada H. A digital computer model of the vectorcardiogram with distance and boundary effects: Simulated myocardial infarction. Am Heart J. 1967;74(6):792-808. doi:10.1016/0002-8703(67)90098-1.

8. Wagner GS, Freye CJ, Palmeri ST, et al. Evaluation of a QRS scoring system for estimating myocardial infarct size. I. Specificity and observer agreement. Circulation. 1982;65(2):342347. doi:10.1161/01.CIR.65.2.342.

9. Strauss DG, Selvester RH. The QRS complex--a biomarker that "images" the heart: QRS scores to quantify myocardial scar in the presence of normal and abnormal ventricular conduction. J Electrocardiol. 2009;42(1):85-96. doi:10.1016/j.jelectrocard.2008.07.011.

10. Stevenson WG, Friedman PL, Sager PT, et al. Exploring Postinfarction Reentrant Ventricular Tachycardia With Entrainment Mapping. J Am Coll Cardiol. 1997;29(6):11801189. doi:10.1016/S0735-1097(97)00065-X.

11. Yokota H, Heidary S, Katikireddy CK, et al. Quantitative characterization of myocardial infarction by cardiovascular magnetic resonance predicts future cardiovascular events in patients with ischemic cardiomyopathy. J Cardiovasc Magn Reson. 2008;10(1):17. doi:10.1186/1532-429X-10-17.

12. Kojodjojo P, Tokuda M, Bohnen M, et al. Electrocardiographic left ventricular scar burden predicts clinical outcomes following infarct-related ventricular tachycardia ablation. Hear Rhythm. 2013;10(8):1119-1124. doi:10.1016/j.hrthm.2013.04.011.

13. Strauss DG, Selvester RH, Lima JAC, et al. ECG Quantification of Myocardial Scar in Cardiomyopathy Patients With or Without Conduction Defects. Circ Arrhythmia Electrophysiol. 2008;1(5).

14. Strauss DG, Mewton N, Verrier RL, et al. Screening entire health system ecg databases to identify patients at increased risk of death. Circ Arrhythmia Electrophysiol. 2013;6(6):11561162. doi:10.1161/CIRCEP.113.000411. 
15. Tanglay $Y$, Twerenbold R, Lee G, et al. Incremental Value of a Single High-sensitivity Cardiac Troponin I Measurement to Rule Out Myocardial Ischemia. Am J Med. 2015;128(6):638-646. doi:10.1016/j.amjmed.2015.01.009.

16. Wagener M, Abächerli R, Honegger U, et al. Diagnostic and Prognostic Value of Lead aVR During Exercise Testing in Patients Suspected of Having Myocardial Ischemia. Am J Cardiol. January 2017. doi:10.1016/j.amjcard.2016.11.056.

17. Buechel RR, Kaufmann BA, Tobler D, Wild D, Zellweger MJ. Non-invasive nuclear myocardial perfusion imaging improves the diagnostic yield of invasive coronary angiography. Eur Heart J Cardiovasc Imaging. 2015;16(8):842-847. doi:10.1093/ehjci/jev095.

18. Zellweger MJ, Maraun M, Osterhues HH, et al. Progression to Overt or Silent CAD in Asymptomatic Patients With Diabetes Mellitus at High Coronary Risk: Main Findings of the Prospective Multicenter BARDOT Trial With a Pilot Randomized Treatment Substudy. JACC Cardiovasc Imaging. 2014;7(10):1001-1010. doi:10.1016/j.jcmg.2014.07.010.

19. Arenja N, Mueller C, EhI NF, et al. Prevalence, extent, and independent predictors of silent myocardial infarction. Am J Med. 2013;126(6):515-522. doi:10.1016/j.amjmed.2012.11.028.

20. Reichlin T, Potocki M, Breidthardt T, et al. Diagnostic and prognostic value of uric acid in patients with acute dyspnea. Am J Med. 2009;122(11):1054.e7-1054.e14. doi:10.1016/j.amjmed.2009.04.023.

21. Breidthardt T, Irfan A, Klima T, et al. Pathophysiology of Lower Extremity Edema in Acute Heart Failure Revisited. Am J Med. 2012;125(11):1124.e1-1124.e8. doi:10.1016/j.amjmed.2011.12.015.

22. Loring Z, Chelliah S, Selvester RH, Wagner G, Strauss DG. A detailed guide for quantification of myocardial scar with the Selvester QRS score in the presence of electrocardiogram confounders. J Electrocardiol. 2011;44(5):544-554. doi:10.1016/j.jelectrocard.2011.06.008.

23. Rosengarten JA, Scott PA, Chiu OKH, Shambrook JS, Curzen NP, Morgan JM. Can QRS scoring predict left ventricular scar and clinical outcomes? Europace. 2013;15(7):1034-1041. doi:10.1093/europace/eut014.

24. Engblom H, Wagner GS, Setser RM, et al. Quantitative clinical assessment of chronic anterior myocardial infarction with delayed enhancement magnetic resonance imaging and QRS scoring. Am Heart J. 2003;146(2):359-366. doi:10.1016/S0002-8703(03)00187-X.

25. Geerse DA, Wu KC, Gorgels AP, Zimmet J, Wagner GS, Miller JM. Comparison between Contrast-Enhanced Magnetic Resonance Imaging and Selvester QRS Scoring System in Estimating Changes in Infarct Size between the Acute and Chronic Phases of Myocardial Infarction. Ann Noninvasive Electrocardiol. 2009;14(4):360-365. doi:10.1111/j.1542474X.2009.00327.x.

26. Strauss DG, Poole JE, Wagner GS, et al. An ECG index of myocardial scar enhances prediction of defibrillator shocks: An analysis of the Sudden Cardiac Death in Heart Failure Trial. Hear Rhythm. 2011;8(1):38-45. doi:10.1016/j.hrthm.2010.09.071.

27. Wieslander B, Nijveldt R, Klem I, et al. Evaluation of Selvester QRS score for use in presence of conduction abnormalities in a broad population. Am Heart J. 2015;170(2):346352. doi:10.1016/j.ahj.2015.05.005.

28. Ward RM, White RD, Ideker RE, et al. Evaluation of a QRS scoring system for estimating myocardial infarct size. Am J Cardiol. 1984;53(6):706-714. doi:10.1016/00029149(84)90390-4. 
29. Weinsaft JW, Kochav JD, Afroz A, Okin PM. Q wave area for stratification of global left ventricular infarct size. Coron Artery Dis. 2014;25(2):138-144.

doi:10.1097/MCA.0000000000000062.

30. Horáček BM, Warren JW, Albano A, et al. Development of an automated Selvester Scoring System for estimating the size of myocardial infarction from the electrocardiogram. $J$ Electrocardiol. 2006;39(2):162-168. doi:10.1016/j.jelectrocard.2005.08.013.

31. Wagner GS, Freye CJ, Palmeri ST, et al. Evaluation of a QRS scoring system for estimating myocardial infarct size. I. Specificity and observer agreement. Circulation. 1982;65(2).

32. Kalogeropoulos AP, Chiladakis JA, Sihlimiris I, Koutsogiannis N, Alexopoulos D.

Predischarge QRS Score and Risk for Heart Failure After First ST-Elevation Myocardial Infarction. J Card Fail. 2008;14(3):225-231. doi:10.1016/j.cardfail.2007.11.004.

33. Abächerli R, Twerenbold R, Boeddinghaus J, et al. Diagnostic and prognostic values of the $V$-index, a novel ECG marker quantifying spatial heterogeneity of ventricular repolarization, in patients with symptoms suggestive of non-ST-elevation myocardial infarction. Int $J$ Cardiol. February 2017. doi:10.1016/j.ijcard.2017.01.151.

34. Wagner A, Mahrholdt H, Holly TA, et al. Contrast-enhanced MRI and routine single photon emission computed tomography (SPECT) perfusion imaging for detection of subendocardial myocardial infarcts: an imaging study. Lancet (London, England). 2003;361(9355):374-379. doi:10.1016/S0140-6736(03)12389-6.

35. Flett AS, Hasleton J, Cook C, et al. Evaluation of techniques for the quantification of myocardial scar of differing etiology using cardiac magnetic resonance. JACC CardiovasC Imaging. 2011;4(2):150-156. doi:10.1016/j.jcmg.2010.11.015.

36. Strauss DG, Selvester RH, Wagner GS. Defining Left Bundle Branch Block in the Era of Cardiac Resynchronization Therapy. Am J Cardiol. 2011;107(6):927-934.

doi:10.1016/j.amjcard.2010.11.010. 


\begin{tabular}{|c|c|c|c|c|}
\hline & & & & \\
\hline & $\begin{array}{l}\text { All patients } \\
\left(\mathrm{n}=2^{\prime} 742\right)\end{array}$ & $\begin{array}{c}<3 \\
\left(n=1^{\prime} 042\right)\end{array}$ & $\begin{array}{c}\geq 3 \\
\left(n=1^{\prime} 700\right)\end{array}$ & $p$-Value \\
\hline Age - years & $68(59-75)$ & $68(60-75)$ & $68(59-76)$ & 0.90 \\
\hline Male gender - no. (\%) & $1938(71)$ & $728(70)$ & $1210(71)$ & 0.49 \\
\hline Risk factors - no. (\%) & & & & \\
\hline Hypertension & $2285(83)$ & $870(83)$ & $1415(83)$ & 0.90 \\
\hline Hypercholesterolemia & $2057(75)$ & $777(75)$ & $1280(75)$ & 0.70 \\
\hline Diabetes & $726(27)$ & $272(26)$ & $454(27)$ & 0.76 \\
\hline Current smoking & $513(19)$ & $180(17)$ & $333(20)$ & 0.15 \\
\hline History of smoking & $1175(43)$ & $453(43)$ & $722(42)$ & 0.63 \\
\hline History - no. (\%) & & & & \\
\hline Coronary artery disease & $1412(51)$ & $504(48)$ & $908(53)$ & 0.01 \\
\hline Previous MI & $890(32)$ & $277(27)$ & $613(36)$ & $<0.001$ \\
\hline Previous revascularization & $1275(46)$ & 459 (44) & $816(48)$ & 0.05 \\
\hline Peripheral artery disease & $285(10)$ & $93(9)$ & $192(11)$ & 0.06 \\
\hline Previous stroke & $249(9)$ & $94(9)$ & $155(9)$ & 0.99 \\
\hline ECG findings - no. (\%) & & & & \\
\hline Q-waves & $318(12)$ & $59(6)$ & $259(15)$ & $<0.001$ \\
\hline LBBB & $94(3)$ & $35(3)$ & $59(3)$ & 0.96 \\
\hline RBBB+LAFB & $60(2)$ & $9(1)$ & $51(3)$ & $<0.001$ \\
\hline RBBB & $89(3)$ & $38(4)$ & $51(3)$ & 0.41 \\
\hline LAFB & $81(3)$ & $4(0)$ & 77 (5) & $<0.001$ \\
\hline LVH & $158(6)$ & $74(7)$ & $84(5)$ & 0.02 \\
\hline No confounders & $2260(82)$ & $882(85)$ & $1378(81)$ & 0.02 \\
\hline Body mass index $\left(\mathrm{kg} / \mathrm{m}^{2}\right)$ & $27[25-31]$ & $27[25-30]$ & $27[25-31]$ & 0.25 \\
\hline MPI Findings & & & & \\
\hline Presence of any scar & $1050(38)$ & $332(32)$ & $718(42)$ & $<0.001$ \\
\hline Presence of $>20 \%$ scar & $164(6)$ & $27(3)$ & $137(8)$ & $<0.001$ \\
\hline $\operatorname{LVEF}(\%)$ & $54[46-63]$ & 56 [49-64] & $53[44-62]$ & $<0.001$ \\
\hline Laboratory findings & & & & \\
\hline hs-cTnT. ng/L & $9[6-16]$ & 9 [5-15] & 10 [6-17] & $<0.001$ \\
\hline Chronic medication & & & & \\
\hline ASA & $1792(65)$ & $683(66)$ & $1109(65)$ & 0.90 \\
\hline Vitamin $\mathrm{K}$ antagonists & $301(11)$ & $94(9)$ & $207(12)$ & 0.01 \\
\hline B-blockers & $1652(60)$ & $611(59)$ & $1041(61)$ & 0.19 \\
\hline Statins & $1718(63)$ & $655(63)$ & $1063(63)$ & 0.89 \\
\hline ACEIs/ARBs & $1740(63)$ & $649(62)$ & $1091(64)$ & 0.34 \\
\hline Calcium antagonists & $671(24)$ & $263(25)$ & $408(24)$ & 0.49 \\
\hline Nitrates & $288(11)$ & $98(9)$ & $190(11)$ & 0.16 \\
\hline
\end{tabular}

${ }^{*} \mathrm{MI}=$ myocardial infarction. $\mathrm{ECG}=$ electrocardiogram. $\mathrm{LBBB}=$ left bundle branch block. $\mathrm{RBBB}=$ right bundle branch block. LAFB = left anterior fascicular block. LVH = left ventricular hypertrophy. $\mathrm{MPI}=$ myocardial perfusion imaging. $\mathrm{LVEF}=$ left ventricular ejection fraction. Hs-cTn $=$ high- 
sensitivity cardiac troponin. $A S A=$ Acetylsalicylic acid. $A C E I S / A R B s=$ angiotensin converting enzyme inhibitor/angiotensin receptor blocker. 


\begin{tabular}{|c|c|c|c|c|}
\hline \multirow[t]{3}{*}{ Table 2} & \multicolumn{4}{|c|}{$\begin{array}{l}\text { Baseline characteristics of the patients with suspected acute } \\
\text { decompensated heart failure (BASEL V) }\end{array}$} \\
\hline & \multirow[b]{2}{*}{ All patients $(n=1151)$} & \multicolumn{2}{|c|}{ QRS-Score } & \multirow[b]{2}{*}{$p$-Value } \\
\hline & & $\begin{array}{c}<3 \\
(n=534)\end{array}$ & $\begin{array}{c}\geq 3 \\
(n=617)\end{array}$ & \\
\hline Age - years & 77 [66. 83] & $76[65.83]$ & $77[66.84]$ & 0.35 \\
\hline Male gender - no. (\%) & $593(52)$ & $261(49)$ & $332(54)$ & 0.12 \\
\hline \multicolumn{5}{|l|}{ Risk factors - no. (\%) } \\
\hline Hypertension & $811(71)$ & $365(68)$ & $446(74)$ & 0.07 \\
\hline Hypercholesterolemia & $397(36)$ & $177(34)$ & $220(38)$ & 0.23 \\
\hline Diabetes & $282(25)$ & $113(21)$ & $169(28)$ & 0.01 \\
\hline Current smoking & $236(21)$ & $108(20)$ & $128(21)$ & 0.90 \\
\hline History of smoking & $511(45)$ & $229(43)$ & $282(46)$ & 0.39 \\
\hline \multicolumn{5}{|l|}{ History - no. (\%) } \\
\hline Coronary artery disease & $415(36)$ & $162(30)$ & $253(42)$ & $<0.001$ \\
\hline Previous MI & $228(21)$ & $84(16)$ & $144(25)$ & 0.001 \\
\hline Previous revascularization & $232(21)$ & $94(18)$ & $138(23)$ & 0.03 \\
\hline Peripheral artery disease & $156(14)$ & $67(13)$ & $89(15)$ & 0.39 \\
\hline Previous stroke & $146(13)$ & $61(11)$ & $85(14)$ & 0.23 \\
\hline \multicolumn{5}{|l|}{ ECG findings - no. (\%) } \\
\hline ST-segment deviation & $57(5)$ & $23(4)$ & $34(6)$ & 0.42 \\
\hline T-wave inversion & $80(7)$ & $44(8)$ & $36(6)$ & 0.14 \\
\hline LBBB & $62(5)$ & $18(3)$ & $44(7)$ & 0.01 \\
\hline RBBB+LAFB & $48(4)$ & $7(1)$ & $41(7)$ & $<0.001$ \\
\hline RBBB & $58(5)$ & $35(7)$ & $23(4)$ & 0.04 \\
\hline LAFB & $39(3)$ & $2(0)$ & $37(6)$ & $<0.001$ \\
\hline LVH & $215(19)$ & $109(20)$ & $106(17)$ & 0.18 \\
\hline Norm & $729(63)$ & $363(68)$ & $366(59)$ & 0.003 \\
\hline \multicolumn{5}{|l|}{$\begin{array}{l}\text { Adjudicated final diagnosis - } \\
\text { no. (\%) }\end{array}$} \\
\hline AHF & $704(61)$ & $292(55)$ & $412(67)$ & $<0.001$ \\
\hline $\begin{array}{l}\text { Non-cardiac causes of } \\
\text { dyspnea* } \\
\text { Imaging Findings - no. (\%) }\end{array}$ & $447(39)$ & $242(45)$ & $205(33)$ & $<0.001$ \\
\hline LVEF & $55[35.60]$ & $55[40.60]$ & $50[34.60]$ & $<0.001$ \\
\hline \multicolumn{5}{|l|}{ Laboratory findings } \\
\hline BNP.ng/l & 476 [117. 1148] & $345[85.857]$ & 605 [159. 1359] & $<0.001$ \\
\hline \multicolumn{5}{|l|}{ Chronic medication - no. (\%) } \\
\hline ASA & $417(37)$ & $192(37)$ & $225(38)$ & 0.73 \\
\hline Vitamin $\mathrm{K}$ antagonists & $302(27)$ & $118(22)$ & $184(31)$ & 0.002 \\
\hline B-blockers & $532(48)$ & $234(45)$ & $298(50)$ & 0.07 \\
\hline Statins & 365 (33) & $170(32)$ & $195(33)$ & 0.88 \\
\hline ACEIs/ARBs & $762(67)$ & 367 (69) & $395(65)$ & 0.11 \\
\hline
\end{tabular}


$\mathrm{MI}=$ myocardial infarction. $\mathrm{ECG}=$ electrocardiogram. $\mathrm{LBBB}=$ left bundle branch block. $\mathrm{RBBB}=$ right bundle branch block. LAFB = left anterior fascicular block. LVH = left ventricular hypertrophy. $\mathrm{MPI}=$ myocardial perfusion imaging. $\mathrm{CHF}=$ congestive heart failure. $\mathrm{LVEF}=$ left ventricular ejection fraction. $B N P=B$-type natriuretic peptide. $A S A=$ Acetylsalicylic acid. $A C E I s / A R B s=$ angiotensin converting enzyme inhibitor/angiotensin receptor blocker.

*Non-cardiac causes of dyspnea included predefined categories such as chronic obstructive pulmonary disease (COPD), asthma bronchiale, pneumonia, bronchitis, Influenza, hyperventilation, pulmonary embolism or cancer. 
Table 3

Cox proportional hazard analysis for all-cause mortality at 360 days

\begin{tabular}{|c|c|c|c|c|c|c|c|c|}
\hline \multirow[b]{3}{*}{ QRS-score $\geq 3$} & \multicolumn{4}{|c|}{ Univariable cox regression } & \multicolumn{4}{|c|}{ Multivariable cox regression } \\
\hline & \multirow{2}{*}{$\begin{array}{c}\text { Hazard ratio } \\
1.48\end{array}$} & \multicolumn{2}{|c|}{$95 \% \mathrm{Cl}$} & \multirow{2}{*}{$\begin{array}{c}\text { p-Value } \\
.001\end{array}$} & \multirow{2}{*}{$\begin{array}{c}\text { Hazard ratio } \\
1.42\end{array}$} & \multicolumn{2}{|c|}{$95 \% \mathrm{Cl}$} & \multirow{2}{*}{$\frac{p-\text { Value }}{.006}$} \\
\hline & & 1.16 & 1.89 & & & 1.10 & 1.83 & \\
\hline Gender (male) & 1.08 & 0.85 & 1.36 & 0.53 & & & & \\
\hline Age $\geq 70$ (years) & 2.90 & 2.10 & 4.02 & $<0.001$ & 2.60 & 1.85 & 3.65 & $<0.001$ \\
\hline Diabetes mellitus & 1.06 & 0.81 & 1.38 & .68 & & & & \\
\hline NYHA III (III vs. II) & 1.97 & 1.11 & 3.51 & .02 & 1.39 & 0.78 & 2.49 & .27 \\
\hline NYHA IV (IV vs. II) & 3.30 & 1.88 & 5.81 & $<0.001$ & 2.37 & 1.34 & 4.20 & 0.003 \\
\hline Atrial Fibrillation & 1.31 & 1.02 & 1.69 & .04 & 0.96 & 0.73 & 1.26 & .77 \\
\hline QRS-width (ms) & 1.30 & 0.99 & 1.70 & .06 & & & & \\
\hline High creatinine ( $\geq 105 \mathrm{~mol} / \mathrm{l})$ & 2.25 & 1.78 & 2.86 & $<0.001$ & 1.96 & 1.53 & 2.52 & $<0.001$ \\
\hline LBBB & 2.15 & 1.45 & 3.20 & $<0.001$ & 1.52 & 0.99 & 2.33 & .05 \\
\hline Heartrate $\geq 80(\mathrm{bpm})$ & 1.63 & 1.24 & 2.14 & $<0.001$ & 1.63 & 1.23 & 2.17 & 0.001 \\
\hline
\end{tabular}

${ }^{*} \mathrm{NYHA}=$ New York Heart Association. LBBB $=$ left bundle branch block. BPM $=$ beats per minute. 
Boxplots for the distribution of the QRS-score according to the extent of myocardial scar on myocardial perfusion imaging.

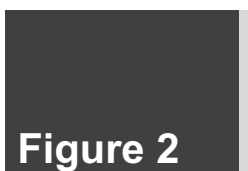

Boxplots for the distribution of the QRS-score according to the left ventricular ejection fraction in (A) the BASEL VIII and in (B) the BASEL V cohort.

Figure 3A

Kaplan-Meier curves for all-cause mortality at $\mathbf{3 6 0}$ days displayed for QRSscore levels $<3$ and $\geq 3$

Figure 3B

Kaplan-Meier curves for cardiovascular re-hospitalization at 360 days displayed for QRS-score levels $<3$ and $\geq 3$ 